\title{
Sur un Thallophyte parasite intestinal de Neodiplogaster rübmi Laumond, 1970 et de Neodiplogaster n.sp. (Nematoda-Rbabditida).
}

\author{
par A. KERMARREC et J.-F. MANIER \\ I.N.R.A. Station de Recherches sur les Nématodes, F. 06 - Antibes \\ Université des Sciences et Techniques du Languedoc ( $\mathrm{P}^{r}$ O. TUZET), F. 34 -Montpellier
}

\begin{abstract}
Résumé
Un parasite mycélien de l'intestin de Neodiplogaster spp., commensaux de Blastophagus piniperda $\mathrm{L}$. et de Pissodes notatus $\mathrm{F}$. est décrit. Des photographies illustrent les différents stades. Ce parasite est trouvé pour la première fois chez les Nématodes; son rapprochement avec un Trichomycète Asellariale: Orchesellaria mauguioi Manier, 1964, est suggéré.
\end{abstract}

\section{Summary}

A mycelian parasite is described from the intestin of Neodiplogaster spp., found in drifts of Blastophagus piniperda L. and Pissodes notatus F., parasitizing Pinus halepensis Mill. of the South of France. Photographs are given of the parasite showing its various stages. This parasite is found for the first time in Nematodes and seems to be very closely related to Orchesellaria mauguioi Manier, 1964 (Trichomycètes Asellariale).

Dans la sciure de galeries imbriquées de Blastophagus piniperda L. et de Pissodes notatus F., coléoptères xylophages parcourant les bois de Pinus halepensis Mill. dans la région de Malpasset (Var), nous avons trouvé, au mois de février 1969, des popu- 
lations importantes de nématodes Rhabditides comprenant: Neodiplogaster rühmi Laumond, 1970, Neodiplogaster n. sp., Macrolaimus canadensis Sanwal, 1960, Meso-

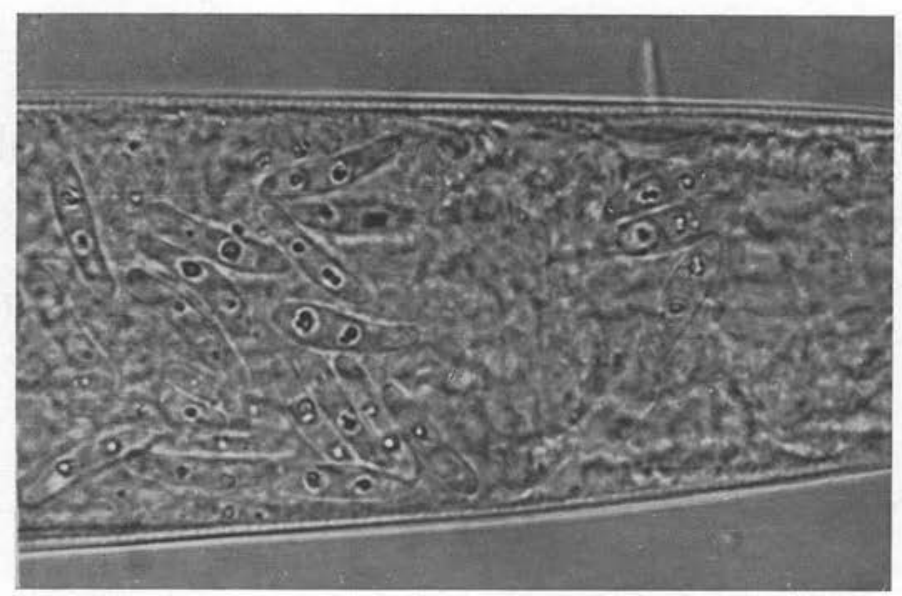

FIG. 1. - Spores dans la zone rectale

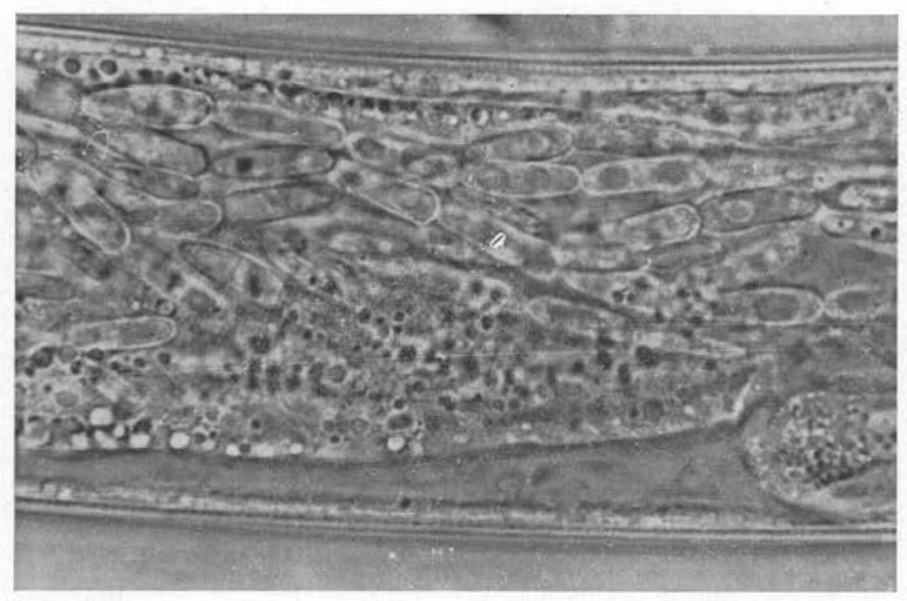

FIG. 2. - Thalle dans l'intestin moyen (zone vulvaire)

diplogaster du groupe lheritieri Maupas, 1919 n. comb., quelques Neotylenchidae et Aphelenchoididae.

Le genre Neodiplogaster y dominait largement et environ $30 \%$ d'entre eux étaient porteurs d'un thallophyte parasite au niveau de la lumière intestinale, du 
cardia au rectum. Neodiplogaster rühmi était sensiblement plus atteint que Neodiplogaster $\mathrm{n}$. sp. (cette dernière espèce est actuellement étudiée sur le plan systématique par C. Laumond).

Le parasite se présente sous deux formes:

$1^{\circ}$ Des éléments isolés libres dans le chyme intestinal près du cardia ou dans la région rectale (fig. 1). Ces cellules uninucléées de 9 à $10 \mu$ de long, 2 à $3 \mu$ de large, plus ou moins cylindriques, légèrement arquées ayant un pôle arrondi, l'autre

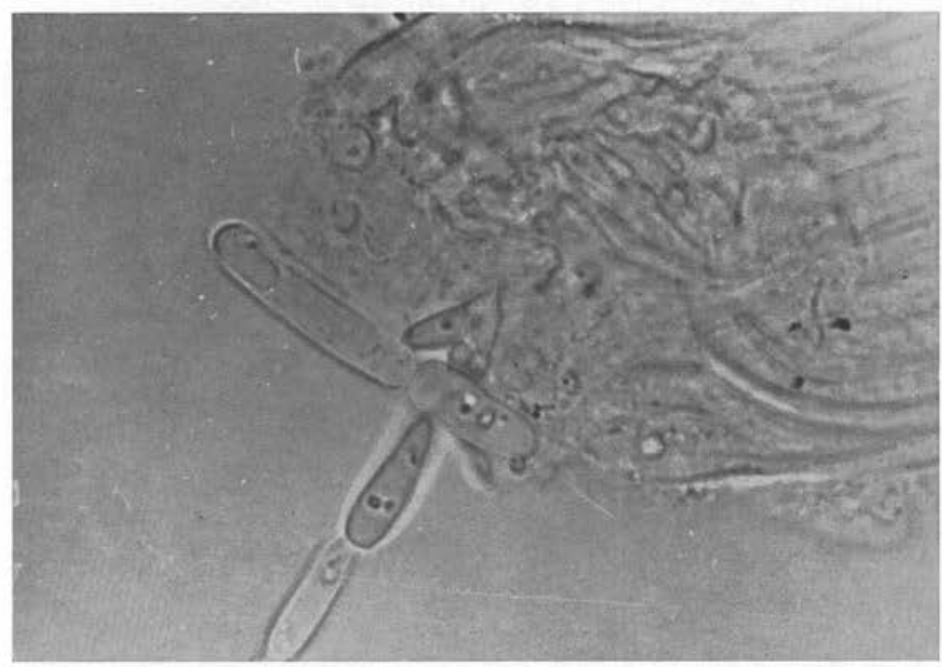

FIG. 3. - Sortie du mycólium après section du corps de $N$. rühmı

plus effilé, doivent être considérées comme étant les agents infestants, c'est-à-dire les spores.

$2^{\circ}$ De longues chaînes ramifiées dérivant des spores par bourgeonnement (fig. 2). Ces hyphes sont constituées de cellules ayant en fin de développement la taille des spores. Elles peuvent s'étendre sur toute la longueur de l'intestin et en remplir tout le volume, causant ainsi une certaine compression des organes pseudocoelomiques parallèles. Par section du corps de l'hôte dans un liquide hypotonique, une partie du thalle parasite est éjectée (fig. 3 et 4). Chaque élément de l'arbuscule tridimentionnel (fig. 5) contient deux inclusions réfringentes animées d'un mouvement complexe de rotation vibrante (fig. 6) et un noyau mis en évidence par la coloration au bleu cotton.

Dans certains animaux, nous avons pu remarquer la présence d'un filament hyalin asepté, relativement long et contourné, moins large que le groupe d'articles 


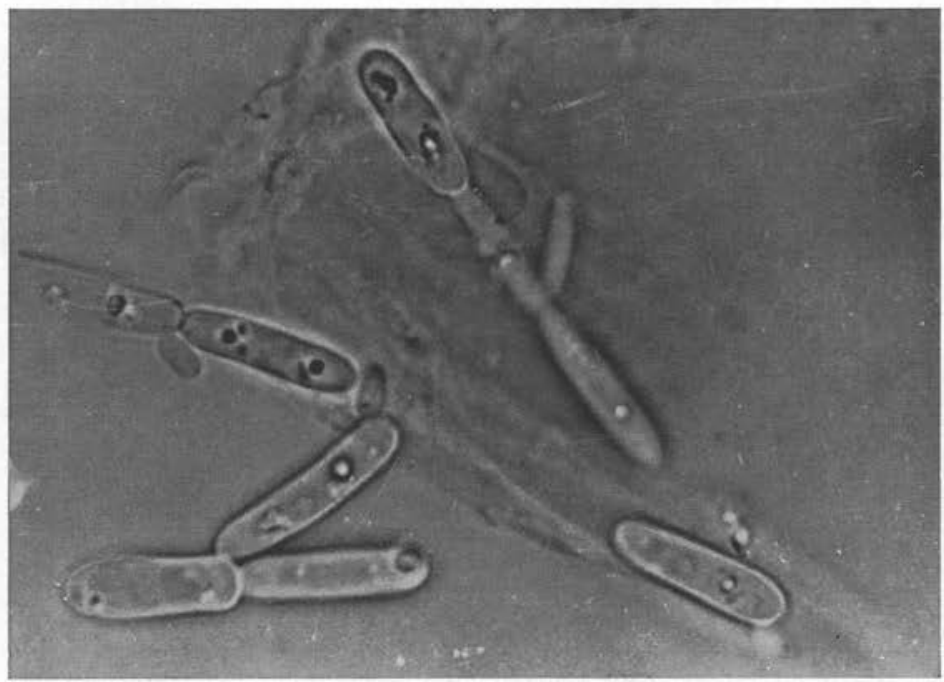

Fig. 4. - Fragments de thalle montrant le bourgeonnement

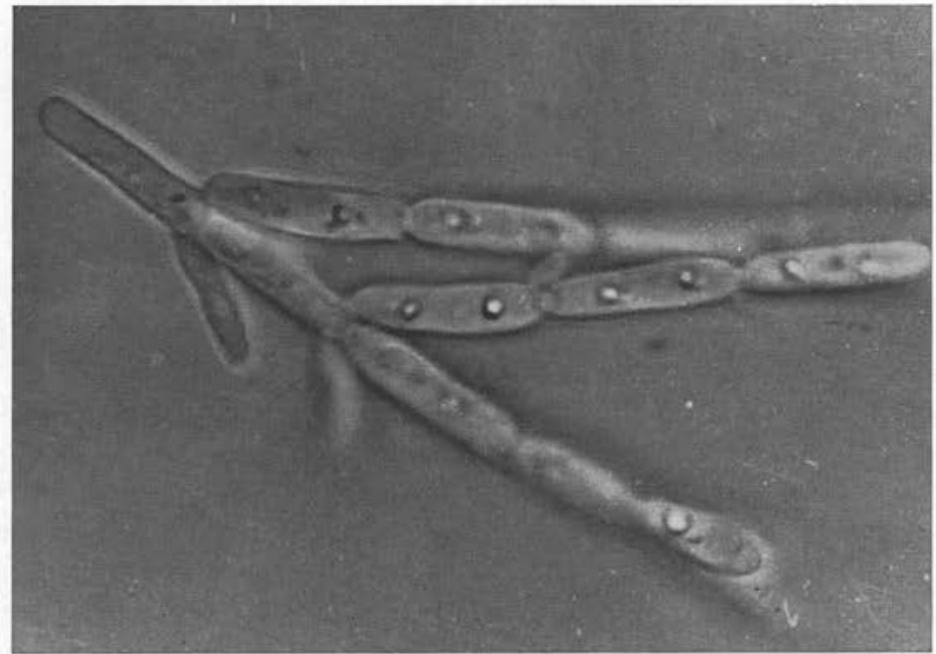

FIG. 5. - Arbuscule extrait de l'intestin 
qu'il prolonge. Ceci correspond probablement à une dégénérescence d'une partie du thalle.

Le parasite n'a été vu que dans le système digestif et seulement chez les adultes où le même taux d'infestation existe dans les deux sexes. Nous avons pu suivre dans le conduit œsophagien d'une femelle parasitée, au niveau de l'isthme, une spore isolée en cours d'ingestion ; elle était aspirée lentement vers le cardia par les mouvements saccadés du bulbe postérieur de l'œsophage. Les larves sont indemnes, car

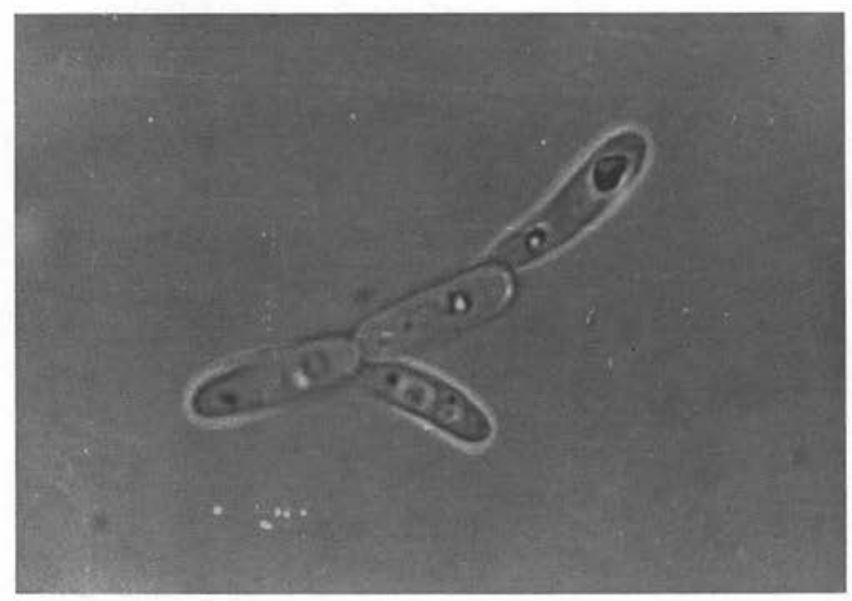

FIG. 6. - Inclusions cytoplasmiques vacuolaires

elles possèdent un stoma étroit et non fonctionnel, c'est en particulier le cas des stades phorétiques de durée ( Dauerlarven »).

Une femelle gravement infestée était gravide: ce type de parasitisme n'entraîne pas de castration parasitaire. Par contre nous avons noté un effet de spoliation nutritive (ou d'encombrement stérique): le contenu intestinal se révélait plus clair en présence de mycélium. Cet endophyte ne semble pas nuire notablement à son hôte.

\section{Cycle.}

Le stade infestant est une spore qui, ingérée par l'adulte, parvient dans la lumière intestinale, se fixe et germe pour donner le thalle qui remplit une partie plus ou moins importante de l'intestin. La formation des spores s'opère par une désarticulation du thalle, libérant de nombreux articles dans la zone rectale, d'où ils sont expulsés dans le milieu extérieur (fig. 7).

Des essais de culture du parasite extrait des nématodes par dissection dans l'eau et repiqué en boîte de Pétri, sur milieu de gélose maltée (15\% agar, $20 \%$ malt) préalablement stérilisé, n'ont abouti qu'à la formation de quelques fragments de thalle peu développés. Le caractère axénique des milieux fut rapidement dénaturé par la 
multiplication de colonies bactériennes et fongiques diverses. Les essais de réinfestation de Neodiplogaster indemnes, suivant le principe de Koch, ont également échoué.

Une seconde campagne de récolte, effectuée à une date semblable dans la région de Malpasset, n'a pas permis une nouvelle mise en évidence de ce parasitisme, ni chez les nématodes, ni chez les insectes colonisant les troncs de pins.

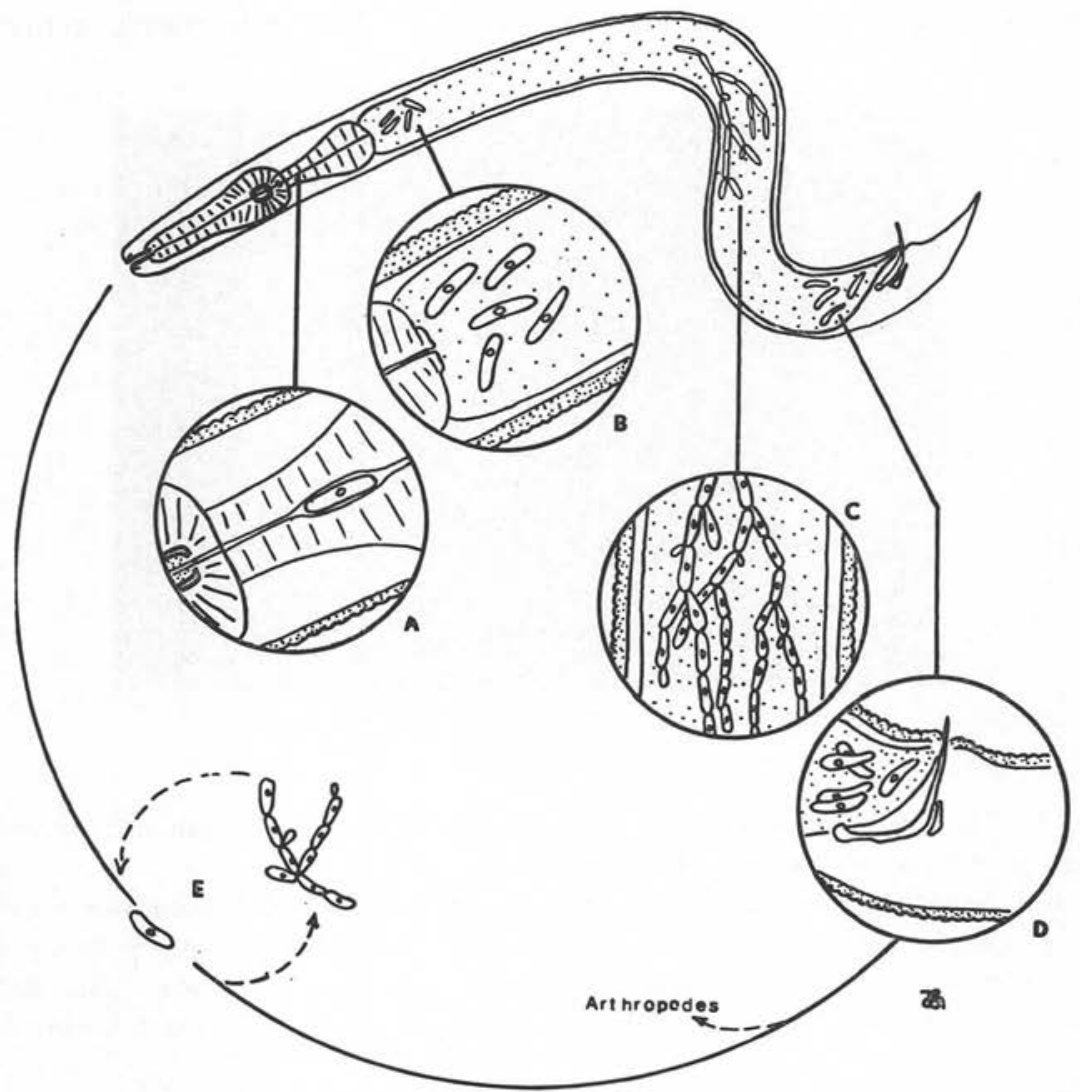

FIG. 7. - Schéma du cyle. - A: phase d'ingestion; B: spores libres dans l'intestin antérieur; C : thalle en formation; D : spores près du rectum; E : phase externe au nématode

\section{Lames.}

Fixation des nématodes en F.A.A. Montage en anneau de paraffine.

$-2 \mathrm{G}$ et $12 \mathrm{G}$ : collection de J.-F. Manier, Laboratoire de Zoologie I, Université des Sciences et Techniques du Languedoc (34) Montpellier.

- 1 G, 3 G, 6 G : I.N.R.A., Station de Recherches sur les Nématodes, (06) Antibes. 


\section{Position systématique du parasite.}

A notre connaissance les endophytes parasitant après ingestion la cavité interne de nématodes sont assez rares. Le mode de pénétration le plus courant reste l'effraction au niveau de la cuticule par une conidie germante ou un hyphe piège au bagage enzymatique suffisant pour perfo:er la paroi de l'hôte. Bütschli (1876) désigne sous le nom de «Schizomycètes » un parasite cœlomique de Tylenchus pelucidus Bast., Metschnikoff (1887) attribue le parasite de Bütschli au genre Monospora Metsch. (levure ascosporée). Lagerheim (1900) note dans les nématodes la présence de Phycomycètes et d'Actinomycétales. L'appartenance des endoparasites signalés par Micoletzky (1922) aux grégarines du groupe des Acephalina reste à confirmer. Dollfus (1946) dans sa revue sur les parasites des helminthes n'ajoute pas de nouveaux exemp'es à ceux déjà signalés.

L'endophyte de l'intestin des Neodiplogaster ne peut être attribué au genre Monospora Metsch. (= Metschnikowia Kam.) bien caractérisé par ses asques à une ou deux spores en aiguille. Sa polarisation semble l'écarter des Saccharomycètes, tandis que sa taille et sa caryo'ogie l'éloigneraient des grèles Actinomycétales. Cet organisme par sa morphologie et son cycle rappelle un Trichomycète Asellariale (1) assez particulier Orchesellaria mauguioi Manier, 1964 du rectum du Collembole Isotomurus palustris Muller. Toutefois l'appartenance du parasite des Neodiplogaster à la classe des Trichomycètes demeure à confirmer en étudiant la dynamique de l'élément initial, sa région basale, le processus de développement de l'endophyte et sa caryologie.

La présence d'Asellariales chez les nématodes n'a jamais été signalée, et constituerait pour cet ordre de Trichomycètes un nouvel aspect de leur parasitisme.

Parmi les Trichomycètes dont la biologie et la systématique ont été étudiées par un des auteurs (Manier, 1969), certains représentants des Eccrinales (Enterobryus, Arthromitus) ont été trouvés sur des nématodes d'insectes (in Dollfus, 1946, p. 247, $\S 56$ à 59). Lorsque des arthropodes hébergent à la fois des Trichomycètes et des nématodes, il est assez fréquent que les premiers se fixent sur les seconds.

\section{Bibliographie}

Butschlı (O.), 1876. - Studien über die ersten Entwickelungsvorgünge der Eizelle, die Zelltheilung und die Conjugation der Infusorien. Abhandlungen herausgeg. von der Senckenberg. Naturforsch. Gesellschaft. Frankfurt am Mein, X, 213-452.

Dollfus (R. P.), 1946. - Parasites (animaux et végétaux) des helminthes. Hyperparasites, ennemis et prédateurs des helminthes parasites et des helminthes libres. Essai de compilation méthodique. Encyclopédie Biologique Lechevalier, Paris, XXVII, 481 pp.

(1) Jusqu'à ce jour (cf. Manier, 1969), tous les Trichomycètes décrits sont parasites d'arthropodes Mandibulates et les Asellariales signalées se développent dans le rectum, soit de crustacés Isopodes, soit d'insectes Collemboles.

Annales de Parasitologie humaine et comparée (Paris), t. 46, $\mathrm{n}^{\circ} 6$ 
Lagerheim (G.), 1900. - Mykologische Studien. III. Beiträge zur Kenntniss der Parasitischen Bacterien und der Bacterioiden Pilze. Bihang till K. Svenska Vet. - Akad. Handl. Stockh. Bd XXVI, Afd 3, $\mathrm{n}^{\circ} 84$, p. 1-21, fig. texte A-G, pl. fig. 1-10.

LAUMOND (C.), 1970. - Un nouveau Neodiplogaster (Nematoda, Diplogasteridae) associé à Pissodes notatus (Coleoptera: Curculionidae). Rev. Zool. Agric. Path. Veg. (à paraître).

Manier (J.-F.), 1964. - Orchesellaria mauguioi n. sp., Trichomycète Asellariale, parasite du rectum de Isotomurus palustris (Muller, 1776), (Insecte Apterygote Collembole), Rev. Ecol. Biol. Sol, 1, 3, 443-449.

-, 1969. - Trichomycètes de France. Annls. Sci. Nat. Bot., 12, X, 565-672.

Metschnikoff (E.), 1884. - Ueber eine Sprosspilzkranheit der Daphnien. Beiträge zur Lehre über den Kampf der Phagocyten gegen Krankheitserreger. Arch. Pathol. Anat. und Physiol., 96, p. 177-195.

Micoletzky (H.), 1922. - Die Freilebenden Erd-Nematoden. Arch. Naturgesch., Berlin, A., $8,9,1-650$. 